\title{
Adsorption of Co(II) from aqueous solution by synthesized chitosan-g-poly (acrylic acid) hydrogel
}

\author{
Qina Sun ${ }^{1, a}$, Yujia Yang ${ }^{1, b}$, Jiawei $\mathrm{Li}^{1, \mathrm{c}}$, Yipeng Shen ${ }^{1, \mathrm{~d}}$ and Junfeng $\mathrm{Li}^{2, \mathrm{e}}$ \\ ${ }^{1}$ School of Environmental and Chemical Engineering, Yanshan University, Qinhuangdao 066004, \\ China; \\ ${ }^{2}$ Institute of Nuclear and New Energy Technology, Tsinghua University, Beijing 100084, China. \\ asunqn@ysu.edu.cn, byujia.Yang@foxmail.com, 474348648@qq.com, dsyp541113@ysu.edu.cn, \\ elijunfeng@mail.tsinghua.edu.cn
}

Keywords: adsorption, chitosan, polymers, hydrogel, Co(II).

\begin{abstract}
Adsorption of $\mathrm{Co}$ (II) on synthesized chitosan- $g$-poly (acrylic acid) hydrogel was investigated, using batch adsorption technique under different experimental conditions of equilibrium $\mathrm{pH}$, concentration of common competing ions, contact time and temperature. The Langmuir adsorption model fitted the experimental data reasonably well better than Freundlich model. The maximum monolayer adsorption capacity of Co(II) was $73.0 \mathrm{mg} / \mathrm{g}$ at $298 \mathrm{~K}$. Kinetics experiments indicated that the adsorption of $\mathrm{Co}$ (II) on synthesized chitosan-g-poly (acrylic acid) hydrogel can be simulated by pseudo-first-order model.
\end{abstract}

\section{Introduction}

With the rapid development of nuclear power application around the world, radioactive wastes containing enormous amounts of radionuclides have become a focus of concern, which may pose serious chemical and radiological toxicity threats to the environment. Among common radioactive nuclides, ${ }^{60} \mathrm{Co}$ is one of the most harmful nuclides owning to its strong $\gamma$-emission and relatively long half-life of 5.3 years [1].

Adsorption removal of ${ }^{60} \mathrm{Co}$ has gained wide attention because of high efficiency, low cost and operation convenience compared with other available removal methods, such as chemical precipitation, reverse osmosis, ion exchange, solvent extraction, coagulation and membrane separation [2, 3]. Chitosan has been investegated with the application for ${ }^{60} \mathrm{Co}$ and other radioactive nuclides adsorption, due to its metal ions adsorption capacity and nontoxicity [4]. As the copolymer obtained by deacetylation of chitin, the hydroxyl and amine groups existing in the chitosan polymer chain can react with radioactive nuclides by the mechanism of chelation and/or ion-exchange [5]. However, the mechanical strength and adsorption rate are often found to be unsatisfied in practice [4].

Hydrogel is the hydrophilic polymers with the structure of three dimensional networks which crosslinked by the chemical or physical interactions [6]. Various functional groups can load on hydrogel, such as amine, carboxylic acid, hydroxyl, and sulfonic acid groups, which attached onto the polymeric networks as the excellent active sites [7-9].

To enhance the mechanical strength and adsorption rate of chitosan absorbent, chitosan based hydrogel was synthesized for simulated radioactive cobolt adsorption in this study. Chitosan was used as the backbone to graft poly (acrylic acid) to synthesize a hydrogel adsorbent chitosan- $g$-poly (acrylic acid) (CTS- $g$-PAA). The effects on $\mathrm{Co}^{2+}$ adsorption were studied in terms of equilibrium $\mathrm{pH}$, concentration of common competing ions, contact time and adsorption temperatures. The adsorption kinetics and isotherms were also investigated.

\section{Material and methods}

Materials. Chitosan (CTS) with 90\% deacetylation degree was provided by Sinopharm Chemical Reagent Co.,Ltd (China). Potassium persulfate (KPS, AR) and N, N'-methylene-bisacrylamide 
(MBA, AR) were provided by Tianjin Kaiton Chemical Reagent Co., Ltd. (China). Acetone (Ac, AR) was provided Tianjin Dongzheng Chemical Industrial Co., Ltd. (China). Acrylic acid (AA, CP), ammonium ferrous sulfate $\left(\left(\mathrm{NH}_{4}\right)_{2} \mathrm{SO}_{4} \cdot \mathrm{FeSO}_{4} \cdot 6 \mathrm{H}_{2} \mathrm{O}, \mathrm{AR}\right)$ and other inorganic reagents were bought from Tianjin Kemiou Chemical Reagent Co., Ltd. (China). All solutions were prepared with deionized water. Solutions of the $\mathrm{NaOH}(0.1 \mathrm{M})$ and the $\mathrm{HNO}_{3}(1 \mathrm{M})$ were used to adjust $\mathrm{pH}$ in bacth adsorption experiments.

Preparation of chitosan-g-poly (acrylic acid) hydrogel. Solution A was obtained by diluting $17.5 \mathrm{~mL}$ AA with $30 \mathrm{~mL}$ deionized water. CTS (1.5g) was completely dissolved in solution A to prepare the colloidal solution B. KPS and ammonium ferrous sulfate were added as initiating agents in colloidal solution B with continual stirring at $70{ }^{\circ} \mathrm{C}$ for $2 \mathrm{~h}$. MBA $(0.1 \mathrm{~g})$ was mixed into the resulting solution and stirred for another $1 \mathrm{~h}$ at the same temperature for obtaining the colloid mixture of CTS and PAA. Then, the colloid mixture of CTS and PAA was rapidly cooled and excessive amount of Ac was added. The resulting mixture was kept in static for $30 \mathrm{~min}$ to prepare the jellied CTS-g-PAA. The jellied CTS-g-PAA was washed by Ac 2 to 3 times and dried for $18-20 \mathrm{~h}$. After that, the prepared material was soaked and washed by deionized water to remove the excessive Ac, dried and grinded to get the CTS-g-PAA adsorbent for the subsequent experiments.

Batch adsorption experiments. In the present study, a series of batch adsorption experiments were used to evaluate adsorption behavior of $\mathrm{Co}^{2+}$ on CTS- $g$-PAA adsorbent. The effect of equilibrium $\mathrm{pH}$, concentration of common competing ions, contact time and temperature were investigated and the adsorption kinetics and isotherms were analyzed and discussed. Stock solutions of $\mathrm{Co}^{2+}, \mathrm{Na}^{+}, \mathrm{Mg}^{2+}$ and $\mathrm{Ca}^{2+}$ ions with the concentration of $1000 \mathrm{mg} / \mathrm{L}$ were prepared by dissolving appropriate amounts of analytical grade nitrate salts in deionized water. Working solution of $15 \mathrm{mg} / \mathrm{L}$ $\mathrm{Co}^{2+}$ was prepared by stepwise dilution of the stock solution. Batch adsorption experiments were carried out in glass flakes with $0.1 \mathrm{~g}$ adsorbent and $50 \mathrm{~mL}$ working solution, and in a temperature-controlled shaker (SHZ-A) with speed of $140 \pm 5 \mathrm{rpm}$ for $24 \mathrm{~h}$. The adsorption temperature was $298 \mathrm{~K}$ in all experiments except for temperature effect experiment, in which a series of 283, 313 and $333 \mathrm{~K}$ were studied. Kinetic experiments were conducted by adding $1.0 \mathrm{~g}$ of CTS- $g$-PAA into $500 \mathrm{~mL} \mathrm{Co}{ }^{2+}$ solution of an initial concentration of $10 \mathrm{mg} / \mathrm{L}$ at $298 \mathrm{~K}$. At various time points, $1.0 \mathrm{~mL}$ solution was taken for $\mathrm{Co}^{2+}$ concentration determination. $\mathrm{Co}^{2+}$ concentration in solutions were analyzed by atomic absorption spectrum method with flame atomization (AA-6800). The equilibrium adsorption capacity $q_{\mathrm{e}}(\mathrm{mg} / \mathrm{g})$ of $\mathrm{Co}^{2+}$ was calculated as follows:

$q_{\mathrm{e}}=\left(C_{0}-C_{\mathrm{e}}\right) V / m$.

Where, $C_{0}$ and $C_{\mathrm{e}}(\mathrm{mg} / \mathrm{L})$ reprent the initial and equilibrium concentration of $\mathrm{Co}^{2+}$ respectively; $V$ is the volume of the $\mathrm{Co}^{2+}$ solution, and $m$ is the weight of CTS-g-PAA.

\section{Results and discussion}

Effects of the solution pH on Co(II) removal. Fig. 1 showed the adsorption efficiency of $\mathrm{Co}^{2+}$ on CTS-g-PAA as a function of $\mathrm{pH}$ ranging from 0.8 to 5.6. The removal was a $\mathrm{pH}$-dependent process with an optimal $\mathrm{pH}$ of approximately 5.5. Lower efficiency was obtained at lower $\mathrm{pH}$ values. Subsequent experiments were performed with the equilibrium $\mathrm{pH}$ around 5.5 in solutions.

Competition with common cations. In radioactive liquid wastes, ${ }^{60} \mathrm{Co}$ coexist with common metal ions. Cations may compete with ${ }^{60} \mathrm{Co}$ during adsorption. Fig. 2 shows the $\mathrm{Co}^{2+}$ removal efficiency in the binary solute systems which contain different molar concentrations of $\mathrm{Na}^{+}, \mathrm{Ca}^{2+}$ and $\mathrm{Mg}^{2+}$. With none competing ions, the adsorption efficiency of $\mathrm{Co}^{2+}$ was $48.05 \%$, marked by the dashed. Adsorption efficiencies in the binary solute systems were reduced in presence of competing ions and the removal efficiency in the $\mathrm{Ca}^{2+} / \mathrm{Co}^{2+}$ system are much lower, which indicated that there existed a competition between $\mathrm{Co}^{2+}$ and coexisting ions for the binding sites and $\mathrm{Ca}^{2+}$ is more affinitive with CTS- $g$-PAA adsorbent.

Effect of temperature and adsorption isotherms. Adsorption isotherms of $\mathrm{Co}^{2+}$ at different temperatures of 298, 313 and $333 \mathrm{~K}$ are presented in Fig.3. Langmuir and Freundlich isotherm models were used to describe experimental data. The equations of two models and equilibrium 
parameters of $\mathrm{Co}^{2+}$ adsorption obtained by regression analysis were listed in Table 1 . Lower temperatures favored $\mathrm{Co}^{2+}$ adsorption on CTS-g-PAA within the experimental ranges, indicating an exothermic adsorption process. Larger $R^{2}$ values of Langmuir isotherm model presented a better correlation than Freundlich model, indicating for monolayer adsorption of $\mathrm{Co}^{2+}$ on CTS- $g$-PAA.

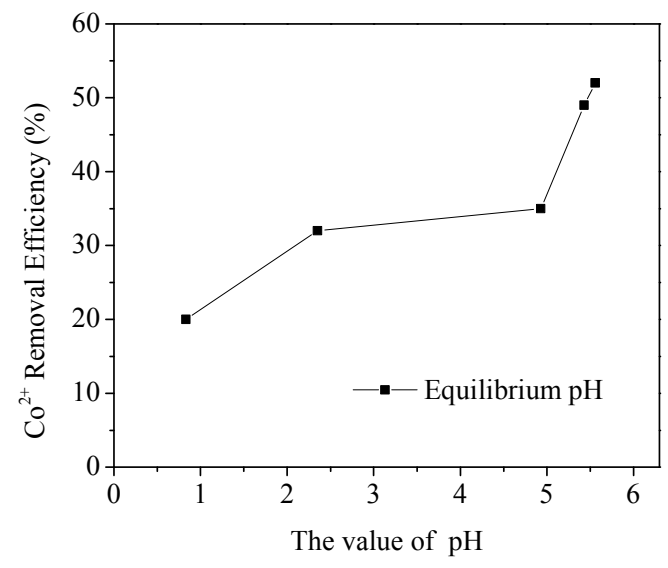

Fig. 1 Effect of $\mathrm{pH}$ on $\mathrm{Co}^{2+}$ removal $(298 \mathrm{~K}$; dose $2 \mathrm{~g} / \mathrm{L}$; initial $\mathrm{Co}^{2+} 15 \mathrm{mg} / \mathrm{L} ; 24 \mathrm{~h}$ )

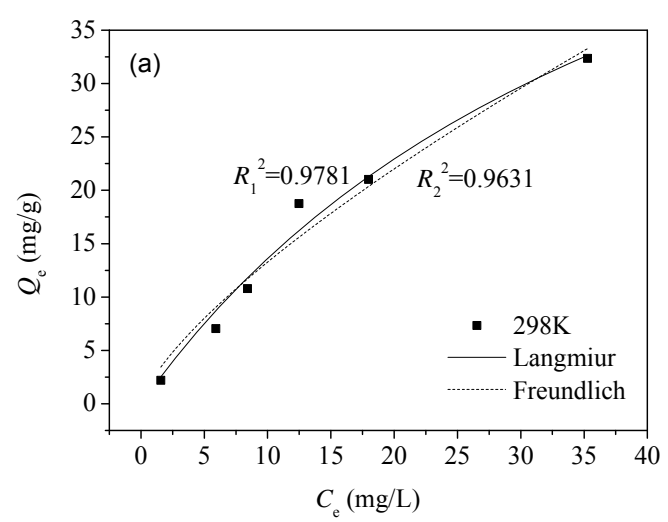

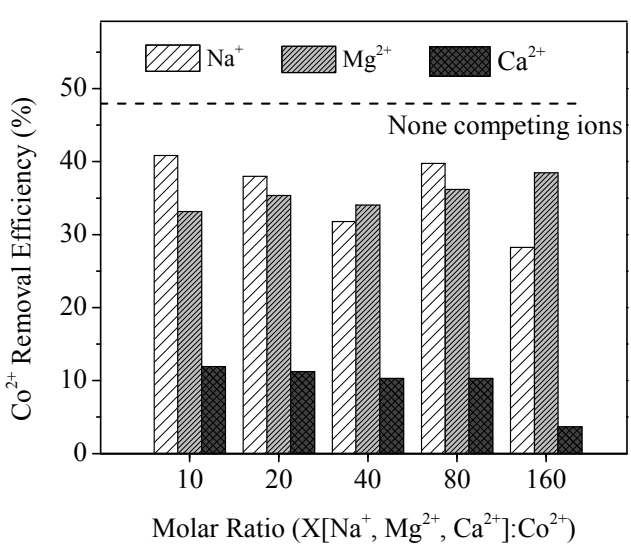

Fig. 2 Effects of competing cations on $\mathrm{Co}^{2+}$ removal $(298 \mathrm{~K}$; adsorbent dose $2 \mathrm{~g} / \mathrm{L}$; initial $\left.\mathrm{Co}^{2+} 15 \mathrm{mg} / \mathrm{L} ; \mathrm{pH} 5.5 ; 24 \mathrm{~h}\right)$

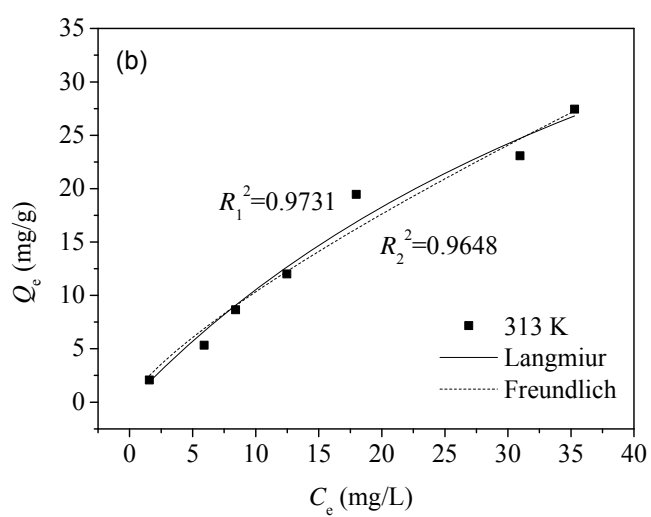

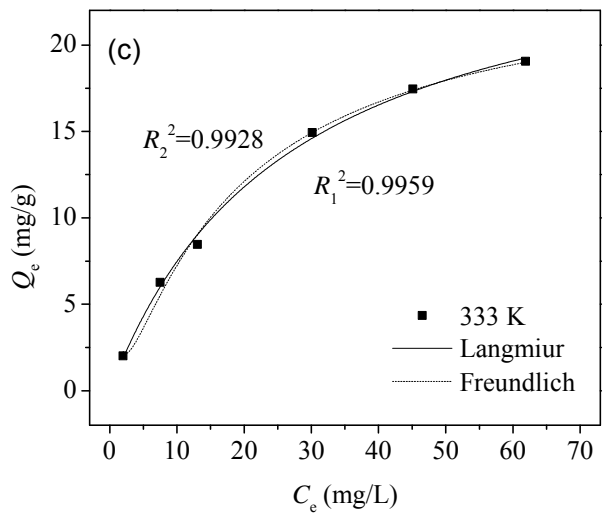

Fig. 3 Adsorption isotherms of $\mathrm{Co}^{2+}$ at different temperatures (dose $2 \mathrm{~g} / \mathrm{L}$; initial $\mathrm{Co}^{2+} 15 \mathrm{mg} / \mathrm{L} ; \mathrm{pH}$ $5.5 ; 24 \mathrm{~h})$

Effect of contact time and adsorption kinetics. Adsorption experiments were carried out at different contact times. Fig. 4 illustrated adsorption capacity of $\mathrm{Co}^{2+}$ consisted of two stages: a primary rapid stage at the first $60 \mathrm{~min}$ and a secondary slow phase from 60 to $240 \mathrm{~min}$. Adsorption equilibrium reached at equilibrium after 100-120 min.

Classic pseudo-first-order and pseudo-second-order models were used to evaluate the kinetic mechanism that controls the $\mathrm{Co}^{2+}$ adsorption on CTS- $g$-PAA. The kinetic models and calculated values of parameters are given in Table 2 , where $q_{t}$ and $q_{\mathrm{e}}$ represent the amount of $\mathrm{Co}^{2+}$ adsorbed 
$(\mathrm{mg} / \mathrm{g})$ at equilibrium and contact time $t$ respectively, $k_{1}\left(\mathrm{~min}^{-1}\right)$ and $k_{2}(\mathrm{~g} /(\mathrm{mg} \cdot \mathrm{min}))$ represent the kinetic rate constants of pseudo-first-order and pseudo-second-order models. The pseudo-first-order model described adsorption data with a higher coefficient of determination of 0.9939 , compared with that of pseudo-second-order model, suggesting a linear relationship between adsorption rate and $\mathrm{Co}^{2+}$ concentration.

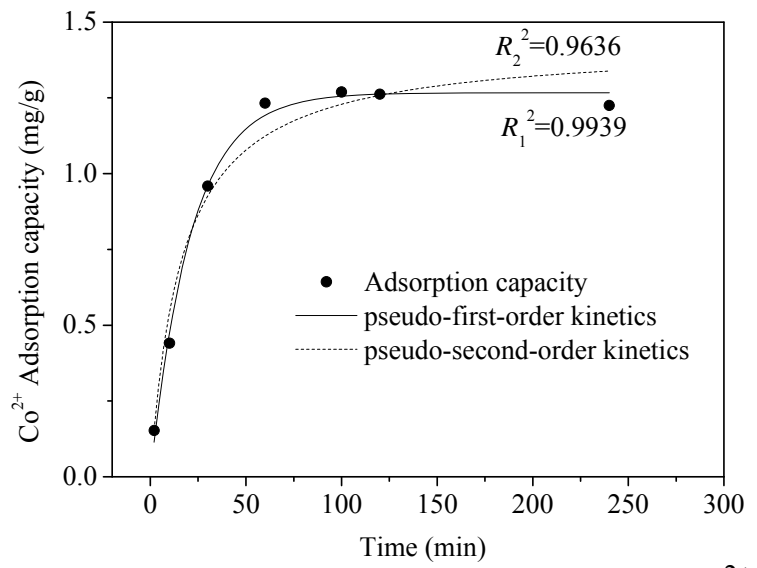

Fig. 4 Adsorption kinetic curve at $298 \mathrm{~K}\left(\right.$ dose $1 \mathrm{~g} / \mathrm{L}$; initial $\mathrm{Co}^{2+} 10 \mathrm{mg} / \mathrm{L} ; \mathrm{pH}=5.5$ )

Table 1 Isotherm models and equilibrium parameters for $\mathrm{Co}^{2+}$ adsorption

\begin{tabular}{cccccc}
\hline \multirow{2}{*}{ Model } & \multirow{2}{*}{ Equation } & \multirow{2}{*}{ Parameter } & \multicolumn{3}{c}{ Temperature (K) } \\
& & $q_{\mathrm{m}}(\mathrm{mg} / \mathrm{g})$, the maximum adsorption capacity & 73.0 & 69.0 & 27.7 \\
Langmuir & $q_{\mathrm{e}}=\frac{q_{m} k_{L} C_{e}}{1+k_{L} C_{e}}$ & $k_{\mathrm{L}}(\mathrm{L} / \mathrm{mg})$, Langmuir constant related to the energy & 0.023 & 0.018 & 0.037 \\
& & $k_{\mathrm{F}}(\mathrm{mg} / \mathrm{g})$, adsorption capacity $(\mathrm{mg} / \mathrm{g})$ & 2.48 & 1.75 & 2.53 \\
Freundlich & $q_{\mathrm{e}}=k_{F} C_{e}{ }^{1 / n}$ & $\mathrm{n}$, Freundlich constant indicative of adsorption & 1.37 & 1.30 & 1.74 \\
\hline
\end{tabular}

Table 2 Kinetic models and parameters for $\mathrm{Co}^{2+}$ adsorption

\begin{tabular}{cccc}
\hline Model & Equation & $q_{\mathrm{e}}$ & $k$ \\
\hline pseudo-first-order & $\log \left(q_{e}-q_{t}\right)=\log q_{e}-\frac{k_{1}}{2.303} t$ & 1.27 & 0.047 \\
pseudo-second-order & $\frac{t}{q_{t}}=\frac{1}{k_{2} q_{e}{ }^{2}}+\frac{t}{q_{e}}$ & 1.43 & 0.043 \\
\hline
\end{tabular}

\section{Summary}

Chitosan- $g$-poly (acrylic acid) hydrogel was synthesized in this study and was expected to present high affinity to $\mathrm{Co}^{2+}$ for the presence of large amounts of functional hydroxyl and amine groups. By the investigation of $\mathrm{Co}^{2+}$ adsorption on CTS- $g$-PAA, this material was domestrated to be a promising adsorbent, in terms of effecting factors adsorption isotherms and kinetics.

\section{Acknowledgements}

This work was financially supported by the National High Technology Research and Development Program of China (Grant No.2012AA063504), the National Natural Science Foundation of China (Grant No. 21301151), the Natural Science Foundation of Hebei Provence (Grant No. B2013203317) and the Science and Technology Research Foundation for Colleges of Hebei Provence (Grant No. QN2015176). 


\section{References}

[1] Y. Park, Y. Lee, W. Shin, S. Choi, Removal of cobalt, strontium and cesium from radioactive laundry wastewater by ammonium molybdophosphate-polyacrylonitrile (AMP-PAN). Chem. Eng. J. 162 (2010) 685-695.

[2] E.H. Borai, M.M. Breky, M.S. Sayed, M.M. Abo-Aly, Synthesis, characterization and application of titanium oxide nanocomposites for removal of radioactive cesium, cobalt and europium ions. J. Colloid Interface Sci. 450 (2015) 17-25.

[3] Vaughan, C. Dieters, W. Fu, K. Byrne, Properties of Lewatit ${ }^{\circledR}$ TP272, a commercial solvent impregnated cation exchange resin for cobalt recovery. Miner. Eng. In press.

[4] T.S. Anirudhan, S. Rijith, A.R. Tharun, Adsorptive removal of thorium(IV) from aqueous solutions using poly(methacrylic acid)-grafted chitosan/bentonite composite matrix: Process design and equilibrium studies. Colloids Surf. A Physicochem Eng. Asp. 368 (2010) 13-22.

[5] M.K. Sureshkumar, D. Das, M.B. Mallia, P.C. Gupta, Adsorption of uranium from aqueous solution using chitosan-tripolyphosphate (CTPP) beads. J. Hazard. Mater. 184 (2010) 65-72.

[6] M. Ahearne, A. Coyle, Application of UVA-riboflavin crosslinking to enhance the mechanical properties of extracellular matrix derived hydrogels. J. Mech. Behav. Biomed. Mater. 54 (2015) 259-267.

[7] Q. Zhu, Z. Li, Hydrogel-supported nanosized hydrous manganese dioxide: Synthesis, characterization, and adsorption behavior study for $\mathrm{Pb}^{2+}, \mathrm{Cu}^{2+}, \mathrm{Cd}^{2+}$ and $\mathrm{Ni}^{2+}$ removal from water. Chem. Eng. J. 281(2015) 69-80.

[8] B. Hui, Y. Zhang, L. Ye, Structure of PVA/gelatin hydrogel beads and adsorption mechanism for advanced $\mathrm{Pb}(\mathrm{II})$ removal. J. Ind. Eng. Chem. 21 (2015) 868-876.

[9] J. Wang, Z. Li, Enhanced selective removal of $\mathrm{Cu}(\mathrm{II})$ from aqueous solution by novel polyethylenimine-functionalized ion imprinted hydrogel: Behaviors and mechanisms. J Hazard Mater 300 (2015) 18-28. 